\title{
PACIENTE ADULTO MAYOR Y CIRUGÍA NO CARDÍACA: ¿QUÉ DEBEMOS SABER?*
}

\author{
Dr. Claudio Nazar J. ${ }^{1}$, Int. Maximiliano Zamora H. ${ }^{2}$, \\ Drs. Ricardo Fuentes H. ${ }^{1}$, Guillermo Lema F. ${ }^{1}$ \\ 1 División de Anestesiología. \\ 2 Interno de Medicina. \\ Escuela de Medicina, Facultad de Medicina, Pontificia Universidad Católica de Chile. \\ Santiago, Chile.
}

\begin{abstract}
Elderly patient and non cardiac surgery: What should we know?

The surgical procedures in elderly patients are and will be more common. They are characterized by greater morbidity and mortality than those in younger patients, so it is essential an appropriate preoperative evaluation, a diligent intraoperative monitoring and a proper management of the postoperative period. The old age has a number of physiological changes and an increased incidence of chronic diseases and together determine a decline in physiological reserve and functional capacity. In the preoperative evaluation is important a systematic assessment of comorbidities and drugs routinely prescribed, "fragility" of these patients due to the surgical procedure and the risk of developing postoperative cognitive deficit. Type of surgery, degree of urgency and patient conditions are the main factors to consider at time to choose type of anesthesia. Common complications include the development of postoperative delirium and postoperative cognitive decline, where efforts should be directed at prevention rather than treatment, because it significantly increases perioperative morbidity.
\end{abstract}

Key words: Surgery, aged, 80 and over, period, perioperative, mortality, morbidity, delirium.

\section{Resumen}

Los procedimientos quirúrgicos en pacientes adultos mayores son cada vez más frecuentes. Se caracterizan por tener mayor morbimortalidad que los realizados en pacientes más jóvenes, por lo que es esencial una apropiada evaluación preoperatoria, una acuciosa monitorización intraoperatoria y un adecuado manejo del período postoperatorio. La edad conlleva una serie de cambios fisiológicos y una mayor incidencia de enfermedades crónicas, que en conjunto determinan una disminución de las reservas fisiológicas y la capacidad funcional. En la evaluación preoperatoria son importantes una valoración exhaustiva de las comorbilidades y fármacos de uso habitual, la posible "fragilidad" de estos pacientes ante el proceso quirúrgico y su riesgo de

*Recibido el 28 de julio de 2014 y aceptado para publicación el 11 de septiembre de 2014.

Los autores no refieren conflictos de interés.

Correspondencia: Dr. Claudio Nazar J.

cenazar@med.puc.cl 
desarrollar déficit cognitivo postoperatorio. El tipo de cirugía y su grado de urgencia, las condiciones actuales del paciente, y las características del centro asistencial son los principales factores a considerar al momento de elegir el tipo de anestesia. Problema frecuente del período postoperatorio es el desarrollo de delirium y deterioro cognitivo, donde los esfuerzos deben dirigirse a su prevención, más que al tratamiento, ya que aumentan considerablemente la morbilidad perioperatoria.

Palabras clave: Cirugía, adulto mayor, período perioperatorio, mortalidad, morbilidad, fragilidad, delirium.

\section{Introducción}

El aumento de la expectativa de vida y la disminución de la natalidad han llevado a un incremento significativo del promedio de edad de la población. Chile sigue esta tendencia, viviendo una etapa avanzada de transición hacia el envejecimiento de su población.

En nuestro país, actualmente una de cada diez personas pertenece al grupo adulto mayor, esperándose que para el año 2025 esta proporción sea de uno cada cinco. La población mayor a 60 años corresponde aproximadamente a dos millones de habitantes, de los cuales más de un cuarto se concentra en personas mayores de 75 años ${ }^{1}$.

Alrededor de un tercio de los pacientes geriátricos se someterán a un procedimiento quirúrgico antes de su muerte ${ }^{2}$, lo que hace necesario conocer detalladamente los aspectos relacionados al manejo perioperatorio de este grupo etario.

La edad por sí misma no debiera ser considerada una contraindicación quirúrgica ni anestésica, ya que el mayor riesgo perioperatorio, está dado principalmente por las eventuales múltiples comorbilidades que el paciente presenta más que por su longevidad. En la evaluación de riesgo perioperatorio, una adecuada clasificación American Society of Anesthesiologists (ASA) es más determinante que la edad cronológica ${ }^{3}$.

En comparación a pacientes jóvenes, los adultos mayores tienen significativamente más morbimortalidad perioperatoria, especialmente en cirugías de urgencia ${ }^{4,5}$, por lo que es prioritario establecer medidas estandarizadas de evaluación y manejo perioperatorios, como valoración preanestésica precoz y completa, monitorización estricta intra y postoperatoria de parámetros vitales, eventual postoperatorio inmediato en unidades de mayor complejidad, etc.

\section{Cambios fisiopatológicos relacionados con la edad}

En la gran mayoría de los adultos mayores existe una disminución de la reserva fisiológica para responder a situaciones de estrés, como una intervención quirúrgica, siendo los sistemas cardiovascular y respiratorio los más afectados por el envejecimiento. Los ancianos presentan con frecuencia disfunción diastólica en grado variable, determinando mayor dependencia del llenado ventricular activo dado por la contracción auricular, siendo más frecuente la aparición de inestabilidad hemodinámica ante arritmias como la fibrilación auricular ${ }^{6}$. Además, presentan mayor frecuencia de cardiopatía coronaria, con el consiguiente mayor riesgo de eventos isquémicos miocárdicos perioperatorios.

La disminución de la masa muscular total y de la elasticidad pulmonar alteran la mecánica ventilatoria, siendo necesario un mayor trabajo respiratorio ${ }^{3}$. También hay una disminución de la respuesta compensatoria ventilatoria ante la hipoxia e hipercapnia, y un aumento de la sensibilidad a los efectos de benzodiacepinas y opioides ${ }^{7}$. Lo anterior lleva a mayor incidencia de depresión respiratoria e hipoventilación en el período postoperatorio. Además, los adultos mayores presentan mayor frecuencia de neumonías aspirativas, atelectasias y edema pulmonar postquirúrgicos ${ }^{8}$. Aproximadamente un $40 \%$ de las muertes de adultos mayores en el período perioperatorio son por complicaciones pulmonares ${ }^{7}$.

Existe disminución fisiológica de la velocidad de filtración glomerular con la edad, que no se ve reflejado en los niveles plasmáticos de creatinina debido a la reducción de la masa muscular. Existe mayor riesgo de insuficiencia renal aguda prerrenal por disminución del volumen circulante efectivo, existiendo también disminución del flujo sanguíneo hepático. La disfunción renal y hepática en ancianos puede llevar a alteraciones del metabolismo y la excreción de diversas drogas utilizadas durante la anestesia, cirugía y período postquirúrgico, las cuales deberían ajustar sus dosis e intervalos de administración ${ }^{6}$.

Existe una significativa menor termorregulación, con el consiguiente mayor riesgo de hipotermia perioperatoria, la que puede provocar eventos adversos como: complicaciones cardiovasculares (isquemia miocárdica, arritmias, crisis hipertensivas), alteraciones de la coagulación, mayor riesgo de infección de herida operatoria, despertar prolongado postanestésico, disconfort termal (calofríos), etc., los cuales aumentan significativamente la morbimortalidad perioperatoria y la estadía hospitalaria ${ }^{9}$. 
La composición corporal cambia, con disminución del porcentaje total de agua corporal y masa magra, y aumento relativo del tejido adiposo ${ }^{10}$. Lo anterior explica porque, utilizando dosis similares, las drogas hidrofílicas tienen menor volumen de distribución y mayor concentración plasmática que en pacientes jóvenes, como el caso de la morfina intravenosa. Lo anterior obliga a ajustar las dosis de este tipo de fármacos en pacientes adultos mayores. Esta alteración farmacológica secundaria al envejecimiento puede verse acentuada por uso crónico de diuréticos, típicamente utilizados en ancianos. Por otro lado, las drogas liposolubles tienen mayor volumen de distribución por el aumento de la grasa corporal, retardando su eliminación, como el caso de los fármacos benzodiacepínicos, los cuales aumentan su vida media de eliminación en adultos mayores.

La disminución del volumen cerebral total con el envejecimiento está asociada a mayor frecuencia de alteraciones cognitivas y demencia, los cuales son factores predictores de delirium postoperatorio.

La Tabla 1 muestra un resumen con los cambios fisiológicos fundamentales en los adultos mayores $^{6,11}$.

\section{Evaluación preoperatoria en pacientes ancianos}

Debe ser exhaustiva en todos los pacientes con el fin de minimizar los posibles riesgos del procedimiento quirúrgico y anestésico, teniendo en cuenta el estado basal del individuo. Esto cobra especial importancia en los pacientes adultos mayores, donde frecuentemente se encuentran comorbilidades (hipertensión arterial, diabetes mellitus, insuficiencia renal, demencia, etc.) y disminución en grados variables de las reservas fisiológicas. Uno de los aspectos más relevantes a evaluar es la capacidad funcional, según criterios de la New York Heart Association (NYHA), la cual refleja directamente su función cardíaca y respiratoria ${ }^{5}$. El reconocimiento de las enfermedades concomitantes de cada paciente, sobre todo las cardiovasculares, pulmonares, hepáticas y renales, es esencial para requerir eventual estudio complementario, solicitar interconsultas a los especialistas respectivos, tomar medidas preventivas y optimizar terapias, disminuyendo así los efectos adversos secundarios propios de los procedimientos quirúrgicos y anestésicos. Se debe evaluar el estado cognitivo de los pacientes adultos mayores, para determinar cuales se encuentran en riesgo de desarrollar delirium y/o disfunción cognitiva postoperatoria, temas que se desarrollarán más adelante. La herramienta más utilizada es el mini-mental state examination, aunque este cuestionario tiene baja sensibilidad para detectar demencia y no descarta disfunción cognitiva ${ }^{3}$.

Hasta un 30\% de los adultos mayores son "frágiles" vamente nuevo y difícil de definir, es un síndrome clínico relacionado con el envejecimiento, caracterizado por falla de los mecanismos que mantienen a los diferentes sistemas fisiológicos funcionando adecuadamente. Fried et al, identificaron las cinco características clínicas más representativas de este síndrome: inactividad, debilidad muscular generali-

Tabla 1. Cambios fundamentales en los adultos mayores ${ }^{6,11}$

\begin{tabular}{|ll|}
\hline Cardiovasculares & - Disfunción diastólica cardíaca \\
& - Alteración autonómica cardiovascular: deterioro del sistema autónomo simpático \\
& - Rigidez de las paredes vasculares \\
& - Mayor riesgo de cardiopatía coronaria \\
Pulmonares & - Disminución de elasticidad pulmonar \\
& - Disminución de la capacidad vital y flujo espiratorio máximo \\
& - Aumento volumen residual y del espacio muerto fisiológico \\
Farmacocinética & - Aumento porcentaje total de tejido adiposo \\
& - Disminución porcentaje total de agua corporal y masa magra muscular \\
Temperatura corporal & - Disminución de la depuración de drogas \\
& - Menor metabolismo basal \\
Sistema nervioso central & - Disminución de la vasoconstricción vascular periférica \\
& - Disminución cantidad total tejido subcutáneo \\
& - Disminución de masa neuronal \\
& - Cambios cognitivos \\
& - Disminución de la memoria de largo plazo \\
\hline
\end{tabular}


zada, baja de peso, fatiga crónica y disminución de la velocidad de la marcha. Con tres o más de estas características se habla de paciente "frágil". La importancia radica en que los pacientes "frágiles" tienen mayor mortalidad en los próximos tres años ${ }^{12}$, lo cual se ve acentuado en procedimientos quirúrgicos e invasivos. La movilización precoz luego de la cirugía y programas de ejercicios estructurados pueden mejorar el estado físico y la fuerza, logrando prevenir parcialmente la fragilidad ${ }^{13}$.

En relación a los exámenes preoperatorios ${ }^{14}$, está demostrado que no sería efectivo realizar estudios de rutina en la mayoría de los adultos mayores, a menos de que tengan alguna comorbilidad que lo justifique ${ }^{6}$. Como se mencionó anteriormente, la edad por sí sola no es indicación de solicitud de exámenes preoperatorios. Sin embargo, se justifica realizar electrocardiograma a los pacientes mayores de 65 años con el fin de identificar una posible comorbilidad cardiovascular severa ${ }^{5,15}$. También hay consenso en medir creatininemia, hematocrito/hemoglobina y electrolitos plasmáticos en los pacientes con edad avanzada (mayores a 65 años), como lo recomienda la ASA y el Ontario Preoperative Testing Group ${ }^{16}$. Solicitar pruebas de coagulación y recuento plaquetario de manera rutinaria no ha demostrado ser efectivo en cambiar la conducta perioperatoria, sin embargo, se deben realizar cuando el paciente tenga manifestaciones clínicas sugerentes de alteraciones de la coagulación y/o tratamiento anticoagulante oral o sistémico ${ }^{17}$. En los pacientes con enfermedad pulmonar obstructiva crónica (EPOC), enfermedad frecuente de encontrar en este subtipo de población, se recomienda solicitar pruebas de función pulmonar, como la espirometría, con el fin de evaluar la alteración pulmonar de base y el componente reversible de un posible patrón obstructivo. De existir este último, se sugiere tratamiento con beta 2 agonistas inhalatorios y corticoides sistémicos previo a la cirugía (ojalá desde $48 \mathrm{~h}$ antes), para disminuir el riesgo de broncoespasmo intraoperatorio y post extubación ${ }^{18}$. La radiografía de tórax no está indicada de forma rutinaria en la evaluación preoperatoria de pacientes adultos mayores, salvo que exista patología pulmonar actual o crónica. La suspensión del hábito tabáquico debe realizarse al menos ocho semanas antes a cualquier intervención quirúrgica o procedimiento invasivo, con el fin de disminuir la morbimortalidad perioperatoria. Se ha visto que este efecto benéfico comienza a producirse a las cuatro semanas de suspendido el tabaco ${ }^{6}$, haciéndose clínicamente significativo a partir de las 8 semanas.

En la Tabla 2, se muestran las recomendaciones descritas por Griffiths et al, en relación a evaluación preoperatoria de los pacientes geriátricos ${ }^{5}$.

\section{Manejo de drogas de uso frecuente en el período preoperatorio}

El consumo de múltiples fármacos en los adultos mayores es frecuente. En Estados Unidos más del $90 \%$ de personas mayores de 65 años consumen al menos un fármaco y $40 \%$ toman 5 o más a la semana ${ }^{19}$. Según la encuesta nacional de salud de Chile, el promedio de fármacos usados por los adultos mayores es de 4,17 medicamentos al día, a diferencia de la población general mayor de 15 años, donde el consumo es $2,65^{20}$. Las comorbilidades más frecuentes de encontrar en la población de adultos mayores sometidos a cirugía son hipertensión arterial, diabetes mellitus y sus complicaciones, por lo que debemos tener especial atención en el tratamiento crónico de estas patologías. Generalmente, los medicamentos de uso crónico tienden a mantenerse en el período perioperatorio, incluso el día de la cirugía, existiendo ciertas excepciones, las cuales se detallan a continuación:

\section{Fármacos antihipertensivos ${ }^{21}$}

- Inhibidores de enzima convertidora de angiotensina y antagonistas del receptor de la angiotensina II son los más frecuentemente utilizados. Estudios muestran aumento de la morbimortalidad postoperatoria relacionado a episodios de hipotensión moderada y severa luego de la inducción anestésica ${ }^{22}$, por lo que estaría recomendado la suspensión de estos fármacos al menos $24 \mathrm{~h}$ previas a la cirugía ${ }^{23}$. Sin embargo, una cuidadosa y adecuada inducción anestésica no debería producir episodios graves de hipotensión, aún en pacientes en los cuales se ha mantenido su terapia antihipertensiva, siendo más importante una acuciosa administración de fármacos anestésicos antes que suspender los fármacos mencionados. Este es un tema que aún se encuentra en discusión por lo que ambas alternativas serían recomendables.

- $\beta$-bloqueadores han demostrado significativa disminución de las complicaciones cardiovasculares postoperatorias, por lo que deben ser mantenidos en los pacientes que los utilizaban previamen$\mathrm{te}^{24,25}$.

- Bloqueadores de canales de calcio tienen evidencia más restringida, y se prefiere mantener en el período preoperatorio ${ }^{23}$.

- Diuréticos deben ser descontinuados el día de la cirugía ${ }^{23}$. 
Tabla 2. Adaptación de recomendaciones sobre evaluación preoperatoria en pacientes geriátricos ${ }^{5}$

\begin{tabular}{|c|c|}
\hline $\begin{array}{l}\text { Antecedentes } \\
\text { médicos y } \\
\text { enfermedad } \\
\text { actual }\end{array}$ & $\begin{array}{l}\text { Comorbilidades } \\
\text { Cardiovascular } \\
\text { a) Evaluar signos vitales } \\
\text { b) Capacidad funcional } \\
\text { c) Solicitar electrocardiograma } \\
\text { Respiratoria } \\
\text { a) Test de función pulmonar en caso de enfermedad pulmonar de base } \\
\text { Hematológica } \\
\text { a) Solicitar hematocrito/hemoglobina } \\
\text { Renal } \\
\text { a) Solicitar creatininemia } \\
\text { b) Electrolitos plasmáticos } \\
\text { Nutricional } \\
\text { a) Peso y talla } \\
\text { b) Índice de masa corporal (IMC) } \\
\text { c) Solicitar albúmina plasmática } \\
\text { Evaluación preanestésica } \\
\text { a) Usos previos de anestesia } \\
\text { b) Evaluación vía aérea } \\
\text { c) Evaluación dentadura (edad avanzada y/o edentados como factores de riesgo para ventilación difícil) } \\
\text { d) Consumo de alcohol, tabaco y drogas } \\
\text { e) Enfermedad actual }\end{array}$ \\
\hline Medicamentos & $\begin{array}{l}\text { a) Medicamentos de uso crónico } \\
\text { b) Uso de terapia anticoagulante (suspensión previa, ¿cuándo?) } \\
\text { c) Alergias relevantes }\end{array}$ \\
\hline Cognitivo & $\begin{array}{l}\text { a) Estado cognitivo de base } \\
\text { b) Factores de riesgo para delirium postoperatorio }\end{array}$ \\
\hline $\begin{array}{l}\text { Capacidad } \\
\text { funcional }\end{array}$ & $\begin{array}{l}\text { a) Escala NYHA } \\
\text { b) Escala de unidad metabólica de reposo (MET) }\end{array}$ \\
\hline $\begin{array}{l}\text { Uso de objetos } \\
\text { funcionales }\end{array}$ & $\begin{array}{l}\text { a) Lentes ópticos } \\
\text { b) Audífonos } \\
\text { c) Silla de rueda } \\
\text { d) Uso de bastón }\end{array}$ \\
\hline
\end{tabular}

\section{Recomendaciones y fármacos para el tratamiento de diabetes mellitus ${ }^{26}$}

En ancianos diabéticos que se someten a cirugías o procedimientos invasivos se debe monitorizar la glucosa plasmática, al menos con hemoglucotest en el período preoperatorio inmediato, siendo recomendable glicemias bajo $140 \mathrm{mg}^{*} \mathrm{dL}^{-1}$ en pacientes electivos y bajo 180 , en caso de urgencias ${ }^{27,28}$.

Fármacos de mayor uso ${ }^{26}$ :

\section{Drogas que aumentan sensibilidad a la insulina}

Biguanidas (metformina), deben suspenderse el día de la cirugía por el riesgo teórico de desarrollar acidosis láctica, que estaría acentuado en adultos mayores por deterioro de la función renal y mayores alteraciones hemodinámicas ${ }^{29}$.

Tiazolidinedionas (pioglitazona y rosiglitazona), pueden causar retención hídrica y edema periférico, recomendándose suspender varios días antes de la cirugía (se desconoce tiempo ideal de suspensión), sugiriéndose interconsulta al especialista.

\section{Drogas que aumentan la secreción de insulina}

Las Sulfonilureas (glipizide y tolbutamida) también deben ser suspendidas el día de la cirugía por el riesgo de desarrollar hipoglicemia.

Las No Sulfonilureas (repaglinida, nateglinide y mitiglinide), deben suspenderse luego de administrada la última dosis, previa ingesta de alimentos. 


\section{Nuevas drogas}

Análogos de GLP-1 (exentide) e inhibidores de DPP-IV (sitagliptina), se pueden administrar el mismo día de la cirugía ya que no producen hipoglicemia.

En caso que el anciano utilice insulina con un esquema basal prandial, cuando sea sometido a un procedimiento que requerirá varias horas de ayuno y de relativa corta duración, se recomienda disminuir la dosis de insulina de acción intermedia/larga la noche anterior en un $20 \%$, para la mañana de la cirugía bajarla en un $50 \%$, suspendiéndose la dosis de insulina rápida ${ }^{30}$. En el caso de que se trate de una cirugía de larga duración es preferible utilizar una infusión de insulina intravenosa, previa interconsulta al diabetólogo.

\section{Fármacos anticoagulantes}

El uso de anticoagulantes orales (ACO) es frecuente. Destacan los antagonistas de vitamina $\mathrm{K}$ $\mathrm{y}$ antiagregantes plaquetarios, como la aspirina, usados profilácticamente en pacientes de alto riesgo cardiovascular y como tratamiento de ciertas arritmias cardíacas.

- Antagonistas de vitamina $K$ : A excepción de que se trate de un procedimiento muy mínimo (como extracción dental u otros odontológicos), se recomienda suspender los antagonistas de vitamina $\mathrm{K}$ (warfarina, acenocoumarol) entre 3 a 5 días previos a la cirugía ${ }^{31,32}$, con la idea de reiniciar los fármacos lo antes posible en el postoperatorio. En pacientes adultos mayores en los que no se han logrado niveles adecuados de INR previo a la cirugía $(\leq 1,4$ en cirugías de alto riesgo de sangrado $\left.^{33}\right)$, se recomienda vitamina $\mathrm{K}$ oral $\mathrm{o}$ intravenosa entre 2,5 y $5 \mathrm{mg}, 12$ a $24 \mathrm{~h}$ previo a la cirugía ${ }^{34}$.

- Heparina: En caso de cambio de terapia con ACO a heparina no fraccionada (HNF) en infusión intravenosa o de bajo peso molecular (HBPM) subcutánea, se sugiere suspenderlas al menos 4 y $24 \mathrm{~h}$ antes de la cirugía, respectivamente, volviendo a administrarlas luego de $24 \mathrm{~h}$ postoperatorias $^{31}$.

- Nuevos anticoagulantes orales (dabigratan o rivaroxaban): sólo se requiere de suspensión $24 \mathrm{~h}$ previo a la cirugía en caso de tener función renal normal, ya que estos actúan inhibiendo la función de factores de la coagulación específicos (Factor Xa principalmente), sin afectar su producción, como en el caso de los antagonistas de vitamina $\mathrm{K}^{32}$.

- Antiagregantes plaquetarios (aspirina y clopidogrel): deben ser suspendidos 7 a 10 días previo al procedimiento quirúrgico ${ }^{32}$.

\section{Elección del tipo de anestesia}

$\mathrm{Al}$ igual que en todos los pacientes, la elección del tipo de anestesia a administrar debe ser basada en el tipo de cirugía, el paciente, las preferencias de éste y del equipo quirúrgico. No existe evidencia sólida de que un tipo específico de anestesia sea la mejor opción para este subgrupo de pacientes ${ }^{5,35}$, por lo que la decisión debe ser caso a caso. Sin embargo, se ha visto que la anestesia regional tiene algunas ventajas como mejor manejo del dolor postoperatorio y menor incidencia de trombosis venosa profunda ${ }^{36}$. Además, hay una disminución de algunos efectos adversos a corto plazo, como hipotensión, delirium postoperatorio, complicaciones cardiovasculares y necesidad de uso de opioides ${ }^{37}$.

Algunas consideraciones específicas de la anestesia general en adulto mayor se resumen en la Tabla $3^{3}$.

\section{Tipos de cirugía}

El riesgo quirúrgico del paciente depende importantemente del tipo de cirugía al que será sometido. En pacientes mayores de 80 años las cirugías más frecuentes son las digestivas, urológicas y traumatológicas. Dentro de las anteriores, destacan por su

Tabla 3. Consideraciones anestésicas relevantes en los adultos mayores en relación a la anestesia general ${ }^{3}$

Anestesia general
Mayor dificultad en ventilación (edentados). Necesidad de
uso de cánulas oro/nasofaríngeas
Reacción hemodinámica exagerada ante laringoscopia. Se
sugiere uso de lidocaína en dosis bajas ( 50 mg IV)
Mayor riesgo de aspiración gástrica
Períodos más prolongados de hipotensión arterial que
aumentan la morbilidad perioperatoria
Exageración de respuesta hipotensora arterial de drogas
como el propofol. Se sugiere reducción de dosis
Prolongación de la eliminación e incremento de la sen-
sibilidad ante benzodiacepinas. Se recomienda reducir
dosis de midazolam en un 50\%. No se recomienda uso de
lorazepam ni diazepam
Anestesia inhalatoria: reducción del MAC (concentración
mínima alveolar) de $6 \%$ por cada década luego de los 20
años. Se sugiere usar concentraciones más bajas
Disminución de la depuración renal de ciertas drogas,
como relajantes musculares. Se sugiere evitar uso de dro-
gas con eliminación renal
Opioides: se sugiere reducción de un $50 \%$ de la dosis de
fentanilo intraoperatorio y morfina parenteral por riesgo
de acumulación de su metabolito activo (morfina 6 glu-
corónido)

\section{Anestesia general}

Mayor dificultad en ventilación (edentados). Necesidad de (n)

Reacción hemodinámica exagerada ante laringoscopia. Se giere uso de lidocaína en dosis bajas (50 mg IV)

Mayor riesgo de aspiración gástrica

Períodos más prolongados de hipotensión arterial que aumentan la morbilidad perioperatoria

Exageración de respuesta hipotensora arterial de drogas como el propofol. Se sugiere reducción de dosis

Prolongación de la eliminación e incremento de la sensibilidad ante benzodiacepinas. Se recomienda reducir is de midazolam en un $50 \%$. No se recomienda uso de

mínima alveolar) de $6 \%$ por cada década luego de los 20 años. Se sugiere usar concentraciones más bajas

Disminución de la depuración renal de ciertas drogas, como relajantes musculares. Se sugiere evitar uso de drogas con eliminación renal

Opioides: se sugiere reducción de un 50\% de la dosis de de acumulación de su metabolito activo (morfina 6 glucorónido) 
alta frecuencia la resección transuretral de la próstata, herniorrafia, colectomía, reemplazo electivo de cadera, cirugía de cataratas y colecistectomía. Como se mencionó anteriormente, los adultos mayores presentan mayor mortalidad postoperatoria en comparación a los pacientes más jóvenes en un mismo tipo de cirugía, lo que se acentúa en cirugías de urgencia. Sin embargo, las cirugías que se realizan con mayor frecuencia tienen baja mortalidad (menor al $2 \%$ ), en especial las urológicas y oculares. La cirugía traumatológica se considera de riesgo intermedio. Los procedimientos quirúrgicos que conllevan el mayor riesgo son las cirugías vasculares, abdominales y torácicas. En general, la cirugía vascular tiene un mayor riesgo perioperatorio por las múltiples comorbilidades que presentan los pacientes sometidos a este tipo de cirugía. Caso particular es el de la colecistectomía, donde la mortalidad aumenta al doble durante los primeros 30 días postcirugía en pacientes sobre ochenta años, comparado con pacientes de menor edad ${ }^{38,39}$.

\section{Problema muy frecuente del período postoperatorio en adultos mayores: Delirium y disfunción cognitiva ${ }^{40}$}

Son complicaciones perioperatorias frecuentes en los adultos mayores, consideradas como un espectro dentro de una misma enfermedad. El delirium postoperatorio (POD) es un trastorno súbito y fluctuante, caracterizado por alteración de la atención, el cual ocurre en las primeras $72 \mathrm{~h}$ postoperatorias ${ }^{41}$. Su incidencia global es aproximadamente $10 \%$, pero en adultos mayores se describen cifras desde 25 y hasta $73 \%$. En Chile, se han identificado cifras de aproximadamente un $40 \%{ }^{42}$. Usualmente existen "gatillantes" para su aparición, destacando el dolor postoperatorio. Los factores de riesgo más importantes para desarrollo de delirium postoperatorio se muestran en la Tabla $4^{41,43}$. La disfunción cognitiva postoperatoria (POCD) es más difícil de caracterizar, siendo un cuadro subagudo que ocurre después de grandes cirugías, especialmente las con gran trauma para el paciente, siendo la pérdida de memoria su principal característica. Los factores de riesgo se sobreponen con los de delirium, siendo la edad avanzada el más importante. Para el diagnóstico de POD existen métodos usados ampliamente en clínica, como el confusion assessment method, el cual requiere que la alteración mental actual del paciente sea aguda y fluctuante, caracterizada por disminución de la atención más alguno de los siguientes criterios: alteración del nivel de conciencia y/o desorganización del pensamiento. Para la POCD no hay criterios bien definidos y no está incluida aún en el Diagnostic and statistical manual of mental disorders $I V$. El tratamiento de ambos se basa en la corrección de los factores desencadenantes, pero lo fundamental sigue siendo la prevención. En el caso del POD, se sugiere evitar el uso de opiáceos, benzodiacepinas, dihidropiridinas y antagonistas del receptor $\mathrm{H}_{1}$ de histamina ${ }^{44}$.

En la Tabla 5 se pueden observar las principales diferencias clínicas entre ambas entidades ${ }^{5,41}$.

Tabla 4. Factores de riesgo y principales gatillantes para delirium postoperatorio y POCD $^{41,43}$

\begin{tabular}{|ll|}
\hline Factores de riesgo & Factores gatillantes \\
Edad avanzada & Dolor postoperatorio \\
Cirugía cardíaca & Hipoxemia \\
Depresión o demencia previa & Hipotensión \\
Polifarmacia & Hipercarbia \\
Privación sensorial (auditiva, visual) & Desórdenes metabólicos (hiponatremia, hipercalcemia, hipoglicemia) \\
& Sepsis \\
& Uso de contención física \\
\hline
\end{tabular}

Tabla 5. Principales diferencias clínicas entre delirium y disfunción cognitiva postoperatoria ${ }^{5,41}$

\begin{tabular}{|lll|}
\hline & Delirium & POCD \\
\hline Aparición & Horas a días & Semanas a meses \\
Inicio & Agudo & Subagudo \\
Duración & Días a semanas & Semanas a meses \\
Atención & Disminuida & Disminuida \\
Conciencia & Alterada con frecuencia & Normal \\
Reversibilidad & Mayoría & Mayoría, pero puede perdurar \\
\hline
\end{tabular}




\section{Conclusiones}

La cirugía y anestesia en mayores de 80 años es cada vez más frecuente, por lo que es importante referirse a los cuidados especiales que requieren este tipo de pacientes. Son múltiples los cambios fisiológicos en los adultos mayores que determinan una disminución de la reserva funcional y una mayor probabilidad de eventos adversos posterior a la cirugía, destacando complicaciones cognitivas, cardiovasculares y respiratorias. Es esencial una adecuada y acuciosa evaluación preoperatoria en estos pacientes, con énfasis en las enfermedades concomitantes, fármacos utilizados, factores de riesgo cardiovascular, respiratorio y mental, entre otros. El manejo global del período perioperatorio, incluyendo tipo de cirugía y anestesia, se debe adaptar a cada adulto mayor en específico.

\section{Referencias}

1. Enfoque estadístico-Adulto Mayor, Boletín informativo del instituto nacional de estadísticas, agosto de 2005.

2. Cook DJ, Rooke GA. Priorities in perioperative geriatrics. Anesth Analg. 2003 Jun;96:1823-36.

3. Miller RD, Pardo M, Stoelting RK. Basics of anesthesia, Capítulo 35 "Elderly Patients". 6th ed. Philadelphia, PA: Elsevier/Saunders; 2011.

4. Li G, Warner M, Lang BH, Huang L, Sun LS. Epidemiology of anesthesia-related mortality in the United States, 1999-2005. Anesthesiology [Research Support, N.I.H., Extramural] 2009;110:759-65.

5. Griffiths R, Beech F, Brown A, Dhesi J, Foo I, Goodall J, et al. Peri-operative care of the elderly 2014: Association of Anaesthetists of Great Britain and Ireland. Anaesthesia 2014;69 Suppl 1:81-98.

6. White PF, White LM, Monk T, Jakobsson J, Raeder J, Mulroy MF, et al. Perioperative care for the older outpatient undergoing ambulatory surgery. Anesth Analg. [Research Support, Non-U.S. Gov't Review] 2012;114:1190-215.

7. Sprung J, Gajic O, Warner DO. Review article: age related alterations in respiratory function-anesthetic considerations. Canadian journal of anaesthesia=Journal canadien d'anesthesie. [Research Support, Non-U.S. Gov't Review] 2006;53:1244-57.

8. Qaseem A, Snow V, Fitterman N, Hornbake ER, Lawrence VA, Smetana GW, et al. Risk assessment for and strategies to reduce perioperative pulmonary complications for patients undergoing noncardiothoracic surgery: a guideline from the American College of Physicians. Annals of internal medicine. [Practice Guideline Research Support, Non-U.S. Gov't] 2006;144:575-80.

9. Sessler DI. Mild perioperative hypothermia. The New England Journal of Medicine 1997;336:1730-7.
10. Rivera R, Antognini JF. Perioperative drug therapy in elderly patients. Anesthesiology 2009;110:1176-81.

11. Corcoran TB, Hillyard S. Cardiopulmonary aspects of anaesthesia for the elderly. Best Pract Res Clin Anaesthesiol. 2011;25:329-54.

12. Fried LP, Tangen CM, Walston J, Newman AB, Hirsch $\mathrm{C}$, Gottdiener J, et al. Fragility in older adults: evidence for a phenotype. The journals of gerontology Series A, Biological sciences and medical sciences. [Research Support, U.S. Gov’t, P.H.S.] 2001;56:M146-56.

13. Palmer RM. Perioperative care of the elderly patient: an update. Cleveland Clinic journal of medicine. [Case Reports] 2009; 76 Suppl 4:S16-21.

14. Nazar JC, Bastidas EJ, Lema FG. Exámenes preoperatorios de rutina en cirugía electiva: ¿Cuál es la evidencia? Rev Chil Cir. 2014;66:188-93.

15. Hepner DL. The role of testing in the preoperative evaluation. Cleveland Clinic journal of medicine. [Case Reports Review]. 2009;76 Suppl 4:S22-7.

16. Benarroch-Gampel J, Sheffield KM, Duncan CB, Brown KM, Han Y, Townsend CM Jr., et al. Preoperative laboratory testing in patients undergoing elective, low-risk ambulatory surgery. Annals of surgery. [Evaluation Studies Research Support, N.I.H., Extramural Research Support, Non-U.S. Gov't] 2012;256:51828.

17. Johansson T, Fritsch G, Flamm M, Hansbauer B, Bachofner N, Mann E, et al. Effectiveness of non-cardiac preoperative testing in non-cardiac elective surgery: a systematic review. Br J Anaesth. [Research Support, Non-U.S. Gov't Review] 2013;110:926-39.

18. Silvanus MT, Groeben H, Peters J. Corticosteroids and inhaled salbutamol in patients with reversible airway obstruction markedly decrease the incidence of bronchospasm after tracheal intubation. Anesthesiology. [Clinical Trial Comparative Study Randomized Controlled Trial] 2004;100:1052-7.

19. Qato DM, Alexander GC, Conti RM, Johnson M, Schumm P, Lindau ST. Use of prescription and overthe-counter medications and dietary supplements among older adults in the United States. JAMA 2008;300:286778.

20. Ministerio de Salud Gobierno de Chile, Facultad de Medicina Pontificia Universidad Católica de Chile, Observatorio Social Universidad Alberto Hurtado. Encuesta nacional de Salud 2009-2010 Tomo I, Consumo de medicamentos en la población, 748 .

21. Nazar JC, Herrera FC, González AA. Manejo preoperatorio de medicamentos en pacientes hipertensos. Rev Chil Cir. 2013;65:267-70.

22. Auron M, Harte B, Kumar A, Michota F. Renin-angiotensin system antagonists in the perioperative setting: clinical consequences and recommendations for practice. Postgrad Med J. 2011;87:472-81.

23. Castanheira L, Fresco P, Macedo AF. Guidelines for the management of chronic medication in the perioperative 
period: systematic review and formal consensus. J Clin Pharm Ther. 2011;36:446-67.

24. Singh A, Antognini JF. Perioperative pharmacology in elderly patients. Curr Opin Anaesthesiol. 2010;23:44954.

25. Lombaard SA, Robbertze R. Perioperative use of beta-blockers in the elderly patient. Anesthesiol Clin. 2009;27:581-97, table of contents.

26. Nazar JC, Herrera FC, González AA. Manejo preoperatorio de pacientes con Diabetes Mellitus. Rev Chil Cir. 2013;65:354-9.

27. American Diabetes A. Executive summary: Standards of medical care in diabetes-2012. Diabetes Care 2012;35 Suppl 1:S4-S10.

28. Bogun M, Inzucchi SE. Inpatient management of diabetes and hyperglycemia. Clin Ther. 2013;35:724-33.

29. Sebranek JJ, Lugli AK, Coursin DB. Glycaemic control in the perioperative period. Br J Anaesth. 2013;111 Suppl 1:118-34.

30. Alexanian SM, McDonnell ME, Akhtar S. Creating a perioperative glycemic control program. Anesthesiol Res Pract. 2011;2011:465974. doi: 10.1155/2011/ 465974. Epub 2011 Sep 6.

31. Douketis JD, Berger PB, Dunn AS, Jaffer AK, Spyropoulos AC, Becker RC, et al. The perioperative management of antithrombotic therapy: American College of Chest Physicians Evidence-Based Clinical Practice Guidelines (8th Edition). Chest. 2008;133(6 Suppl):299S-339S.

32. Ortel TL. Perioperative management of patients on chronic antithrombotic therapy. Hematology Am Soc Hematol Educ Program. 2012;2012:529-35.

33. Manchikanti L, Falco FJ, Benyamin RM, Caraway DL, Kaye AD, Helm S, 2nd, et al. Assessment of bleeding risk of interventional techniques: a best evidence synthesis of practice patterns and perioperative management of anticoagulant and antithrombotic therapy. Pain physician. [Review]. 2013;16(2 Suppl):SE261-318.

34. Lubetsky A, Yonath H, Olchovsky D, Loebstein R, Halkin H, Ezra D. Comparison of oral vs intravenous phytonadione (vitamin K1) in patients with excessive anticoagulation: a prospective randomized controlled study. Archives of internal medicine. [Clinical Trial Comparative Study Randomized Controlled Trial]
2003; 163:2469-73.

35. Carlisle JB. Pre-operative co-morbidity and postoperative survival in the elderly: beyond one lunar orbit. Anaesthesia [Review] 2014;69 Suppl 1:17-25.

36. Rodgers A, Walker N, Schug S, McKee A, Kehlet $\mathrm{H}$, van Zundert A, et al. Reduction of postoperative mortality and morbidity with epidural or spinal anaesthesia: results from overview of randomised trials. Bmj. [Research Support, Non-U.S. Gov’t Review] 2000;321(7275):1493.

37. Memtsoudis SG, Sun X, Chiu YL, Stundner O, Liu SS, Banerjee S, et al. Perioperative comparative effectiveness of anesthetic technique in orthopedic patients. Anesthesiology [Comparative Study Research Support, N.I.H., Extramural] 2013;118:1046-58.

38. Vaid S, Bell T, Grim R, Ahuja V. Predicting risk of death in general surgery patients on the basis of preoperative variables using American College of Surgeons National Surgical Quality Improvement Program data. The Permanente journal 2012 Fall;16:10-7.

39. Hamel MB, Henderson WG, Khuri SF, Daley J. Surgical outcomes for patients aged 80 and older: morbidity and mortality from major noncardiac surgery. Journal of the American Geriatrics Society. [Multicenter Study Research Support, Non-U.S. Gov't] 2005;53:424-9.

40. Vega PE, Nazar JC, Rattalino FM, Pedemonte TJ, Carrasco GM. Delirium postoperatorio: una consecuencia del envejecimiento poblacional. Rev Med Chile 2014;142:481-93.

41. Krenk L, Rasmussen LS. Postoperative delirium and postoperative cognitive dysfunction in the elderly-what are the differences? Minerva Anestesiol. 2011;77:742-9.

42. García N, Fuentes R, Rodríguez J, Ramírez G, Sievers P. Factores de riesgo para el desarrollo de delirium postoperatorio en pacientes adultos mayores: estudio clínico prospectivo analítico. Rev Chil Anest. 2013;42:157-61.

43. Mantz J, Hemmings HC, Jr, Boddaert J. Case scenario: postoperative delirium in elderly surgical patients. Anesthesiology 2010;112:189-95.

44. Strom C, Rasmussen LS, Sieber FE. Should general anaesthesia be avoided in the elderly? Anaesthesia [Research Support, N.I.H., Extramural Research Support, Non-U.S. Gov’t Review] 2014;69 Suppl 1:35-44. 